\title{
A Novel Fault Diagnosis Approach for Rolling Bearing Based on CWT and Adaptive Sparse Representation
}

\author{
Xing Yuan (D), Huijie Zhang ${ }^{D}$, and Hui Liu \\ State Key Laboratory for Manufacturing System Engineering, Xi'an Jiaotong University, Xi'an, China \\ Correspondence should be addressed to Huijie Zhang; zhanghuijie@xjtu.edu.cn
}

Received 12 October 2021; Accepted 21 December 2021; Published 1 March 2022

Academic Editor: Gang Tang

Copyright (c) 2022 Xing Yuan et al. This is an open access article distributed under the Creative Commons Attribution License, which permits unrestricted use, distribution, and reproduction in any medium, provided the original work is properly cited.

\begin{abstract}
Extraction and enhancement of weak impulse signature is the key of rolling bearing fault prognostics in which case the features are often weak and covered by noise. Tunable Q-factor wavelet transform (TQWT), as an emerging wavelet construction theory developed in a frequency domain explicitly, has the advantages of matching with the specific oscillation behavior of signal components. In this article, an adaptive sparse representation (ASR) method is proposed, which integrates the sparse code shrinkage (SCS) and parameter optimization into TQWT. However, direct application of ASR is difficult to extract fault signatures at the early stage or low-speed operation due to weak fault symptoms and background noise. A novel fault diagnosis strategy based on continuous wavelet transform (CWT) and ASR is investigated. CWT owns significant advantages on multiscale subdivision and weak signal detection. The results of simulated and experimental vibration signal analyses verify the effectiveness of the proposed method in accurately extracting weak impulse features from the noise environment.
\end{abstract}

\section{Introduction}

Rolling bearings are key components in the mechanical industry, such as wind turbine, warship, aeroengine, and high-end machine tools. During the last three decades, the numbers of usage in rolling bearing systems have substantially increased in both defense and commercial applications. Accounting with the increase in higher operating speed and larger carried load, premature failures due to material fatigue occur in bearing systems. Such premature failures in the bearing systems are always subjected to losses in time and finance, or even sometimes, they may lead to catastrophic consequences. Fault detection has an important role associated with mechanical equipment. This could be a benefit if a fault in components is detected at its early stage, so corrective action can be taken promptly. Presently, the prevention and management of premature failures in equipment has become a vital part of the maintenance program $[1,2]$. However, how to diagnose the rolling bearing faults as early as possible to prevent severe or catastrophic accidents is still a challenge in the diagnosis field. There are many reasons for this problem, including the complexity of bearing contact motion, weak dynamic response, aliasing variability, multisource coupling, mechanical structure filtering, and service condition uncertainty.

Localized defects are often initiated by insufficient lubrication film between the surfaces that are in contact or normal fatigue failure, and the defects grow in size and change shape over time. It is one of the most reliable and sensitive technical means for bearing diagnosis to extract fault features through vibration response collected by an acceleration sensor. When the inner race, outer race, or rolling element of rolling bearing is damaged, a periodic mechanical impulse will occur during the contact process between the fault surface and the other component surfaces, which will excite the natural frequencies of the inner race and outer race, and the periodic transient impulse signals often appear in the vibration signals [3]. Therefore, the extraction and analysis of a periodic impulse signal is the key problem to rolling bearing fault diagnosis. To reliably detect bearing faults, advanced signal processing technology has to 
be used [4-9]. The main purpose of these methods is to extract fault features. Periodic impulse features, especially at early stage and low-speed operation, are often seriously covered by background noise and various disturbances, which bring challenges to fault feature extraction. Signal purification (i.e., noise suppression and interference elimination) methods are hence required to improve the fault detection ability.

Wavelet analysis is the research hotspot in the field of mechanical fault diagnosis for many years, due to its flexible time-frequency resolution and excellent capability of detecting transients [10]. Lin used nonorthogonal Morlet wavelet as the basis function and applied CWT in mechanical fault diagnosis, which was proved to be effective $[11,12]$. Recently, Silva applied CWT to early singlepoint rub detection in a gas turbine and demonstrated that CWT is sensitive to an outbreak of rub [13]. Compared with the classical method of constructing wavelet basis function directly in the time domain, the theory of constructing wavelet from in the frequency domain is developing rapidly. Tunable Q-factor wavelet transform (TQWT), originally proposed by Selesnick, can tune the transform according to the oscillation behavior of the applied signal and has the advantage of fast implementation using radix-2 FFTs [14]. Later, Luo and He provided a deep and detailed analysis of TQWT including the filter bank, decomposition level, $Q$ factor, and parameter influence $[15,16]$. Based on TQWT, Selesnick further proposed resonance-based sparse signal decomposition (RSSD) [17], which decomposes the high resonance and low resonance components according to the signal resonance properties. Cai studied the nonlinear decomposition method based on signal oscillation property and proposed redundant dictionary construction based on TQWT for the sparse representation of gearbox vibration signal [18, 19]. Zhang and Yu studied the multifault diagnosis of gearbox based on RSSD and comb filter [20].

Nevertheless, there is still room to improve further on the TQWT analysis concept and to bring it better in line with other state-of-the-art vibration analysis methods. In this article, a new adaptive sparse representation (ASR) method is proposed, which can well match the damped oscillation mode of the bearing fault signal and enhance the transient impulse characteristics. For the adaptive parameter selection, we use the envelope spectrum fault frequency ratio method to ensure that the fault information is not lost. This paper attempts to highlight the utility of sparsity from the TQWT-SCS perspective and reiterates the effectiveness of the CWT regarding the weak signal detection for the pretreatment step. Meanwhile, for the optimal scale selection of CWT, we propose the strategy of weighted Shannon entropy. The main contributions of this paper are in two categories. We propose a new ASR method for bearings derived from the TQWT, known as TQWT-SCS. We also demonstrate that ASR can handle noise and enhance impulse signals. Moreover, a novel fault diagnosis approach for rolling bearing based on CWT and TQWT-SCS is investigated, which enriches the application of a hybrid wavelet in mechanical diagnosis.
The remainder of this paper is organized as follows. In Section 2, the CWT theories are reviewed and optimal scale selection is discussed. Section 3 introduces TQWT theory, and the proposed ASR method is illustrated in detail. In Section 4, a simulation experiment is given to verify the reliability of the proposed method. Section 5 provides more detailed results of experimental signal processing. Finally, some conclusions are presented in Section 6.

\section{Continuous Wavelet Transform}

The wavelet is obtained by a single function $\psi(t)$ through translation and expansion [21]:

$$
\psi_{a, b}(t)=\frac{1}{\sqrt{a}} \psi\left(\frac{t-b}{a}\right),
$$

where $a$ is the so-called scaling parameter and $b$ is the time localization parameter. The CWT of a finite energy signal $x(t)$ with the analyzing wavelet $\psi(t)$ is defined as the inner product in the Hilbert space:

$$
\begin{aligned}
W T_{x}(a, b) & =\frac{1}{\sqrt{a}} \int x(t) \psi^{*}\left(\frac{t-b}{a}\right) d t \\
& =\int x(t) \psi_{a, b}^{*}(t) d t \\
& =\left\langle x(t), \psi_{a, b}(t)\right\rangle .
\end{aligned}
$$

To effectively reveal the characteristic components of the vibration signal, it is necessary to select the appropriate basis wavelet. At this stage, although there is not a standard method to select the basis wavelet for different tasks, we can choose a proper wavelet according to practical signal characteristic. The rolling bearing fault signal has the remarkable property of periodic transient impulse, and the Morlet wavelet function is very similar to the impulse signal, so it is comparatively suitable for selecting it to extract an impulse feature. Generally, the real part of the Morlet wavelet is used as the base wavelet in real signal processing. The Morlet wavelet is defined as

$$
\psi(t)=e^{-t^{2} / 2} \cos (\mu \pi t),
$$

where $\mu$ is the parameter adjustment factor.

CWT can analyze signal in multiple scales, which can describe the local detailed features in different scales through translation. As the reconstruction of different scale signals lead to different classification results, this research presents a new optimal reconstruction scale determination method based on weighted Shannon entropy. Shannon entropy is a well-adopted sparse measure, which is defined as

$$
E(P)=-\sum_{1}^{n} P_{i} \log P_{i},
$$

where $P_{i}$ is the probability of observing the possible value of a random variable. 
On the one hand, although Shannon entropy can be shown to be a good measure of randomness and sparseness, it is difficult to distinguish the periodic harmonic components in the bearing test signal. On the other hand, the kurtosis value $K$ is sensitive to the outliers or impulses generated by mechanical fault because it is proportional to the fourth-order higher moment. Therefore, considering both Shannon entropy and kurtosis would provide a more reliable scale selection process. The weighted Shannon entropy is defined as

$$
E K=\frac{E}{K} .
$$

Then, the best scale can be found by the minimum value of $E K$. The scale selected by $E K$ is considered to contain the signal nature closest to the impulses produced by the mechanical fault. This selection method can thus reduce the side effects of noise and unrelated signals from periodic harmonic components.

\section{Adaptive Sparse Representation}

3.1. Tunable Q-Factor Wavelet Transform. As one of the powerful tools to analyze the bearing vibration signal, the CWT can effectively identify the periodic impulse features caused by local faults. Nevertheless, due to the influence of the vibration transmission path and interfering noise, the periodic impulse is still very weak, which makes it difficult to effectively extract the fault features. Consequently, a new adaptive sparse representation method based on TQWTSCS is proposed to handle the reconstructed signal after CWT preprocessing containing the periodic impulses, further highlighting the impulse features and improving the accuracy of fault identification.

The difference between TQWT and classic dyadic wavelet transform is that TQWT realizes $Q$-factor adjustment and redundant operation by iterating filter banks through the low-pass scale factor $\alpha$ and high-pass scale factor $\beta$ [14], as shown in Figure 1. This design concept can not only extract the oscillation mode but also obtain the detailed characteristics, which is very suitable for matching the attenuation oscillation mode of the rolling bearing fault signal.

TQWT directly designates quality factor $Q$ and redundancy factor $r$ to design wavelet, which further increases the flexibility of quality factor selection and makes wavelet acquisition more convenient. After $Q$ and $r$ are selected, $\alpha$ and $\beta$ can be obtained by

$$
\begin{aligned}
& \beta=\frac{2}{1+Q}, \\
& \alpha=1-\frac{\beta}{r} .
\end{aligned}
$$

The expressions of low-pass filter and high-pass filter are as follows:

$$
\begin{aligned}
& \left\{\begin{array}{l}
L_{0}(\omega)=1,|\omega| \leq(1-\beta) \pi, \\
L_{0}(\omega)=\theta\left(\frac{\omega+(\beta-1) \pi}{\alpha+\beta-1}\right),(1-\beta) \pi<|\omega|<\alpha \pi, \\
L_{0}(\omega)=0, \alpha \pi \leq|\omega| \leq \pi,
\end{array}\right. \\
& \left\{\begin{array}{l}
G_{0}(\omega)=0,|\omega| \leq(1-\beta) \pi, \\
G_{0}(\omega)=\theta\left(\frac{\alpha \pi-\omega}{\alpha+\beta-1}\right),(1-\beta) \pi<|\omega|<\alpha \pi, \\
G_{0}(\omega)=1, \alpha \pi \leq|\omega| \leq \pi,
\end{array}\right.
\end{aligned}
$$

where $\theta(\omega)=0.5(1+\cos \omega) \sqrt{2-\cos \omega},|\omega| \leq \pi$.

Furthermore, the decomposition level $J$ is limited by the length of the signal $N$. Their relation can be expressed as

$$
J \leq \frac{\log (\beta N / 8)}{\log (1 / \alpha)} .
$$

When $0<\alpha \leq 1$, the input signal is set as $X(\omega)$, and the low-scale filtering characteristic of the corresponding output signal $Y(\omega)$ is expressed as follows:

$$
Y(\omega)=X(\alpha \omega),|\omega| \leq \pi
$$

If $\alpha \geq 1$,

$$
Y(\omega)=\left\{\begin{array}{l}
X(\alpha \omega), \quad|\omega| \leq \pi / \alpha, \\
0, \quad \pi / \alpha<|\omega| \leq \pi .
\end{array}\right.
$$

For the high scale, when $0<\beta \leq 1$, the filtering characteristic is given by

$$
Y(\omega)= \begin{cases}X(\beta \omega+(1-\beta) \pi), & 0<\omega<\pi, \\ X(\beta \omega-(1-\beta) \pi), & -\pi<\omega<0 .\end{cases}
$$

If $\beta \geq 1$,

$$
Y(\omega)=\left\{\begin{array}{l}
X(\beta \omega+(1-\beta) \pi),\left(1-\frac{1}{\beta}\right) \pi<\omega<\pi, \\
X(\beta \omega-(1-\beta) \pi),-\pi<\omega<-\left(1-\frac{1}{\beta}\right) \pi, \\
0,|\omega|<\left(1-\frac{1}{\beta}\right) \pi .
\end{array}\right.
$$

According to the frequency domain characteristics of high-low-pass filter and its subsequent high-low-pass scale transformation, combining with the iterative operation of the basic filter banks under the multiscale decomposition, 


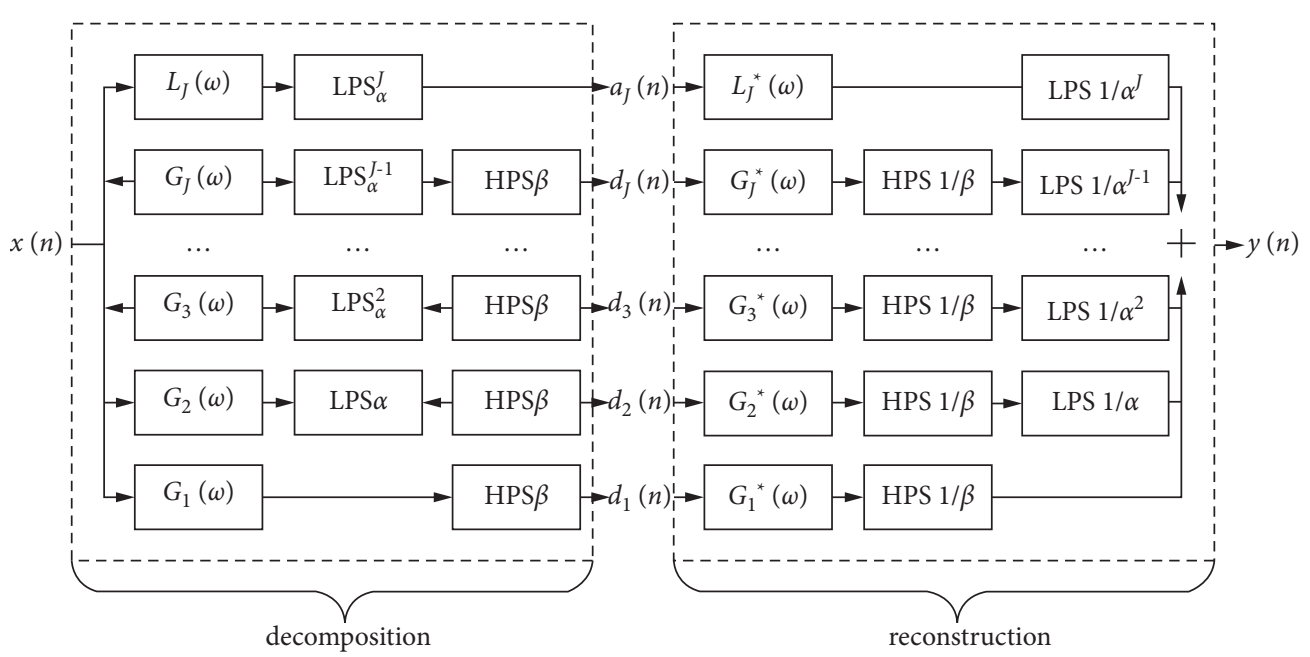

Figure 1: The decomposition and reconstruction process of TQWT.

the equivalent frequency response function for the $J^{\text {th }}$ stage is given by

$$
\begin{aligned}
& L_{j}(\omega)=\left\{\begin{array}{l}
\prod_{m=0}^{j-1} L_{0}\left(\frac{\omega}{\alpha^{m}}\right), \quad|\omega| \leq \alpha^{j} \pi, \\
0, \quad \alpha^{j} \pi<|\omega|<\pi,
\end{array}\right. \\
& G_{j}(\omega)=\left\{\begin{array}{l}
G_{0}\left(\frac{\omega}{\alpha^{j-1}}\right) \prod_{m=0}^{j-2} L_{0}\left(\frac{\omega}{\alpha^{m}}\right), \quad(1-\beta) \alpha^{j-1} \pi<|\omega| \leq \alpha^{j-1} \pi, \\
0, \quad|\omega| \leq \pi .
\end{array}\right.
\end{aligned}
$$

3.2. Sparse Code Shrinkage. The SCS algorithm [22] proposed by Hyvarinen uses the statistical characteristics of non-Gaussian components to get the threshold shrinkage function and denoises the measured signal by removing the noise with the same or similar frequency as the target signal. The sparse representation of a bearing vibration signal can be realized by the SCS algorithm because the fault-generated impulse is strongly non-Gaussian in statistical characteristics. Actually, the local damage vibration signal of rolling bearing is characterized by the nonGaussian property, repetitive transient impulse waveform, and typical sparseness.

As we assume $x_{f}$ is the original fault signal and $v$ is the Gaussian noise of zero mean and variance $\sigma^{2}$, then the observed signal $x$ is given by

$$
x=x_{f}+v .
$$

To represent a sparse distribution, Hyvarinen proposed the following probability density function of a sparse signal [22]:

$$
p\left(x_{f}\right)=\frac{1}{2 d} \frac{(\alpha+2)[\alpha(\alpha+1) / 2]^{(\alpha / 2+1)}}{\left[\sqrt{\alpha(\alpha+1) / 2}+\left|x_{f} / d\right|\right]^{(\alpha+3)}}
$$

where $d$ is the standard deviation of the fault impulse signal $x_{f}$ and $\alpha$ is the parameter controlling the sparseness of the probability density function. In this article, we set $\alpha=0.1$ to adapt the bearing fault feature extraction [11].

For the sparse signal with this distribution, the following thresholding rule can be obtained according to the maximum likelihood principle [22]:

$$
\tilde{x}_{f}=\operatorname{sign}(x) \max \left(0, \frac{|x|-a d}{2}+\frac{1}{2} \sqrt{(|x|+a d)^{2}-4 \sigma^{2}(\alpha+3)}\right) \text {, }
$$

where $a=\sqrt{\alpha(\alpha+1) / 2}, \sigma$ is the standard deviation of the noise, and $x_{f}$ is set to zero in the case that the square root in the above equation is imaginary. The SCS algorithm is used as TQWT threshold, and the noise standard deviation is estimated for each scale $[23,24]$ :

$$
\sigma=\frac{\operatorname{mad}}{0.6745}
$$

where mad denotes the median absolute deviation of wavelet coefficients.

As we assume $x$ is the zero mean, for $x$ and $v$ are uncorrelated, the standard deviation $d$ of $x_{f}$ can be estimated by $[23,24]$

$$
d=\sqrt{\sigma_{x}^{2}-\sigma^{2}}
$$

It is worth noting that the standard deviation $d$ needs to be calculated separately on each wavelet scale.

3.3. Adaptive Sparse Representation. As a new explicit wavelet construction theory in the frequency domain, TQWT has the advantages of matching the specific oscillation behavior of signal components and fast implementation by the FFT algorithm. SCS is a useful tool for signal sparse representation. Naturally, the TQWT along with SCS can efficiently implement the multiscale sparse decomposition of dynamic signals. 
For TQWT, as mentioned before, three parameters need to be determined: $Q$-factor, decomposition level $J$, and redundancy $r$. The specified value of the redundancy must satisfy that $r>1$. In this work, the redundancy $r$ is set to 3 by considering the translation invariance and complex calculations [14]. The decomposition level $J$ only affects the frequency domain decomposition performance in the low-frequency region. Too many decomposition levels will lead to high computational cost and may lead to an excessive decomposition or redundant decomposition of fault characteristic frequency band information. The selection of $J$ should meet the requirements of formula (4). $Q$ is an important parameter, which directly affects the extent of wavelet sustained oscillation. We know that the bearing fault signal has local attenuation oscillation behavior. Therefore, the selection of $Q$ has an important influence on matching the bearing fault signal. To adaptively determine $Q$ and $J$, we use the fault characteristic frequency ratio (FR) of the envelope spectrum to measure the sparse representation effect of TQWT-SCS. $\mathrm{FR}$ is formulated as

$$
F R=\frac{\sum F A\left(k f_{c}\right)}{\sum F A(f)} .
$$

Here, FR is the ratio of fault characteristic frequency to the total amplitude of envelope spectrum. $f_{c}$ is the fault characteristic frequency of rolling bearing. Generally, the first three characteristic frequencies are taken, as $k=3$. The four characteristic frequencies (cage frequency, outer race fault frequency, inner race fault frequency, and ball fault frequency) are listed as follows:

$$
\begin{aligned}
& \text { Cage frequency: } f_{\text {cage }}=f_{r} / 2\left(1-D_{b} / D_{p} \cos \beta\right) \\
& \text { Outer race fault frequency: } f o=Z f_{\text {cage }} \\
& \text { Inner race fault frequency: } f i=f_{r} / 2\left(1+D_{b} / D_{p} \cos \beta\right) \\
& \text { Ball fault frequency: } \quad f b=D_{p} / D_{b}\left[1-\left(D_{b} / D_{p}\right)^{2}\right. \\
& \left.(\cos \beta)^{2}\right] f_{r}
\end{aligned}
$$

where $f_{r}$ is the shaft rotation frequency, $D_{b}$ is the roller diameter, $D_{p}$ is the pitch circle diameter, $\beta$ is the contact angle, and $Z$ is the number of rolling elements.

It should be noted that the fault bearing can not only induce impulse characteristics but also produce a vibration signal having statistical properties that vary cyclically with time. The identification of cyclostationarity allows the separation of different mechanical sources. In addition, the description parameters of cyclostationarity carry useful information about specific mechanical subsets of diagnostic equipment. Cyclostationarity plays an important role in the rolling bearing fault diagnosis since it has been demonstrated that the vibration signals are well modeled by cyclostationary processes $[25,26]$. Cyclostationary coupling sparse diagnosis is also a topic worthy of research in the future.

\section{Simulation Validation}

The schematic diagram of the proposed method is illustrated in Figure 2.
To validate the feasibility of the proposed method for extracting weak impulse signals, a simulation experiment was carried out. In the experiment, the vibration response of bearing with local damage is simulated by an equal-interval impulse signal. The impulse signal is polluted by a lot of white noise and periodic signals. The simulated signal can be expressed as

$$
\begin{aligned}
x(t) & =H(t)+s(t)+n(t), \\
H(t) & =\sum_{k} A_{k} h\left(t-1 / f-\lambda_{i}\right), \\
h(t) & =e^{-40 t} \sin (2 \pi 500 t), \\
s(t) & =\sin (2 \pi 15 t)+\sin (2 \pi 30 t), \\
n(t) & =\operatorname{rand}(n) .
\end{aligned}
$$

Here, $f=10 \mathrm{~Hz}$ is an impulse interval frequency. $A_{k}$ and $\lambda_{i}$, respectively, represent the amplitude and phase of the impulse signal, and the sampling frequency is set to $2000 \mathrm{~Hz}$.

Figures 3(a) and 3(b) display the periodic impulse signal and envelope spectrum. Figure 3(c) and Figure 3(d) show the mixed signal and envelope spectrum, respectively. It can be seen from the figure that the periodic impulse signal is covered by periodic components and noise, and the impulse characteristics cannot be seen. Meanwhile, the main characteristic spectral lines are also swamped by noise in the envelope spectrum; most of which are weak. Hence, it becomes very difficult to distinguish impulses from noise components. We extract the impulse characteristics according to the flow in Figure 2. As shown in Figures 3(e) and $3(\mathrm{f})$, the proposed approach $(Q=3.3$ and $J=9)$ preserves much of the signal magnitude and makes it much easier to identify the true impulses. The sparseness of the signal impulse is effectively enhanced and is becoming more observable. The main characteristic frequencies in the envelope spectrum are also very significant. In fact, this is very important in fault diagnosis.

\section{Method Verification-Case Studies}

5.1. Case Study 1. The bearing vibration data come from Case Western Reserve University [27], while Figure 4 depicts the experimental test bench. The test bearings support the motor shaft. Single point faults were introduced to the deep groove ball bearings (SKF 6205) using electrodischarge machining with different fault diameters. Vibration data were collected using accelerometers at a sampling frequency of 12,000 samples/s. Each bearing was tested under four different loads of $0,1,2$, and $3 \mathrm{hp}$. In this research, we employ the outer race and inner race fault data with the rotating speed 1797 and the fault diameter 0.007 .

Figure 5 shows the outer race fault signal and its envelope spectrum, from which we can see that the impulse signal is obvious, and the spectrum also shows the fault frequency fo $(107.36 \mathrm{~Hz})$ and its harmonic components $2 f_{0}$, $3 f o$, and $4 f o$. However, the sideband component of the outer race fault is relatively weak, which has been well reflected after the application of the proposed approach $(Q=4.3$ and 


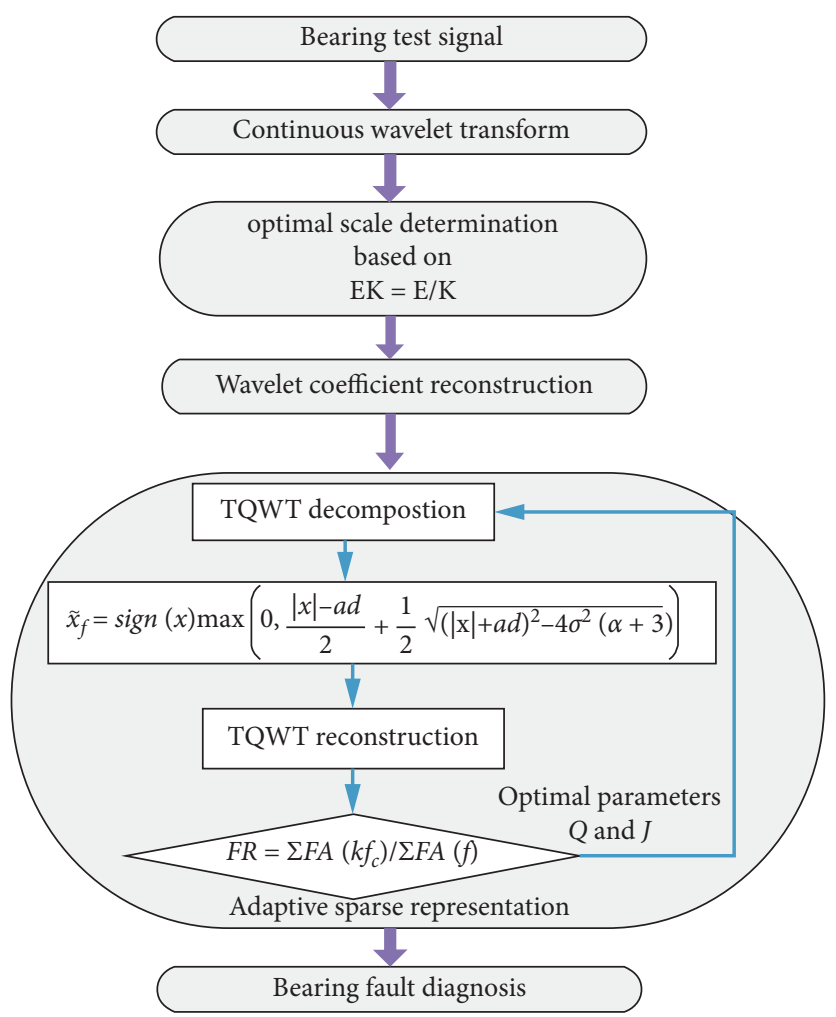

FIGURE 2: Illustration of the proposed approach.

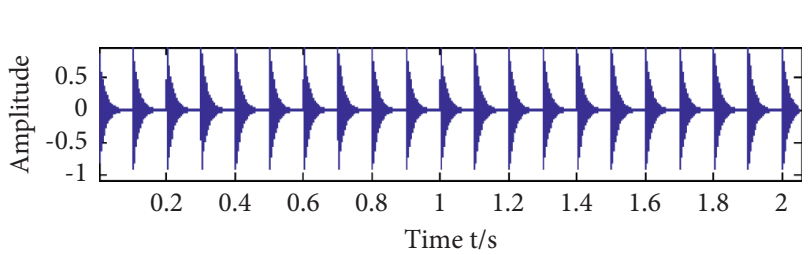

(a)

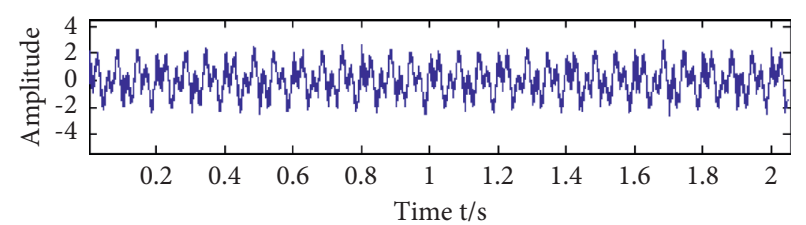

(c)

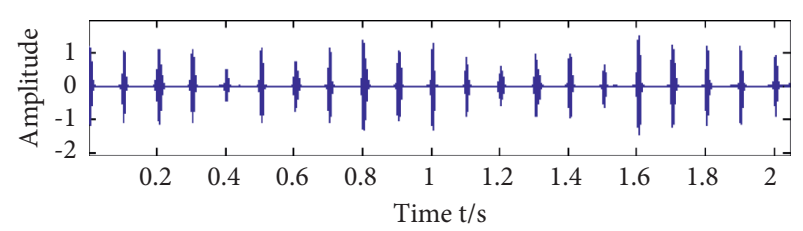

(e)

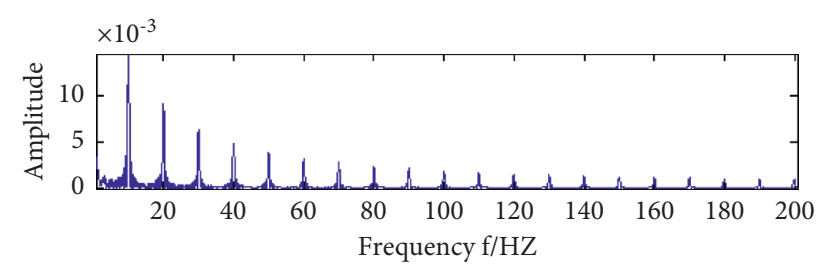

(b)

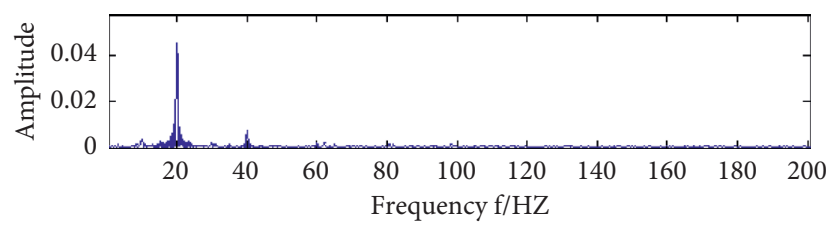

(d)

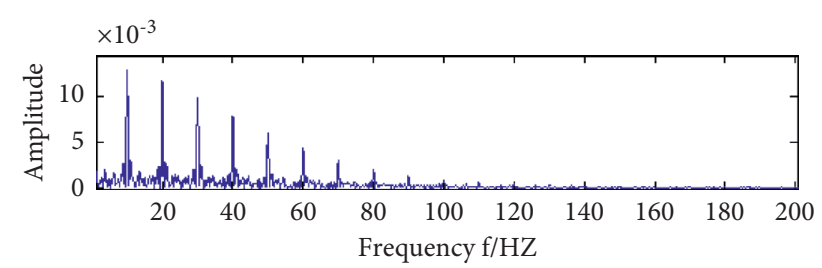

(f)

FIgURe 3: Simulation results: (a) periodic impulse signal; (b) envelope spectrum of periodic impulse signal; (c) mixed signal; (d) envelope spectrum of mixed signal; (e) extracted signal by proposed method; (f) envelope spectrum of extracted signal.

$J=10$ ). It can be seen from Figure 6 that the impulse characteristics are very evident after sparse processing, and the envelope spectrum shows the fault frequency, and its modulation sideband very well. Similar to the outer race fault signal, for the inner race fault signal with low-intensity noise, the impulses can be seen easily with bare eyes 


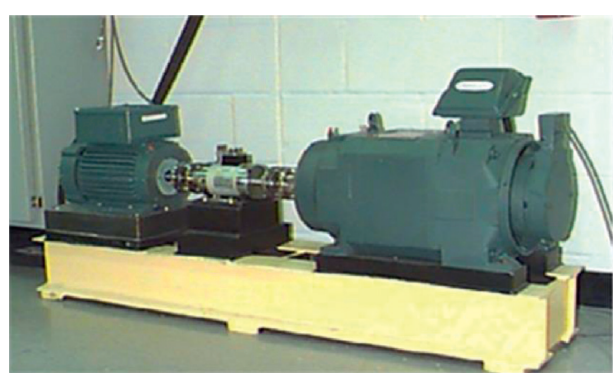

Figure 4: The experimental test apparatus.

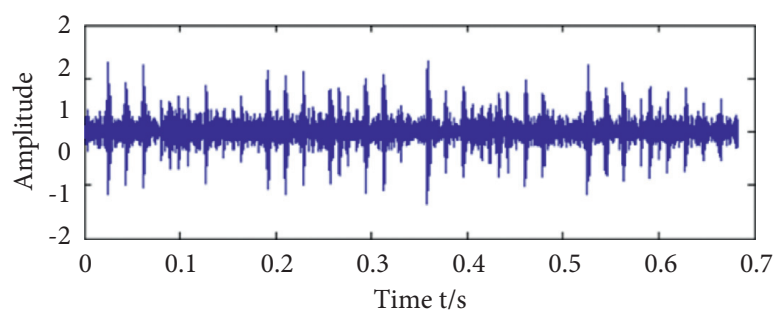

(a)

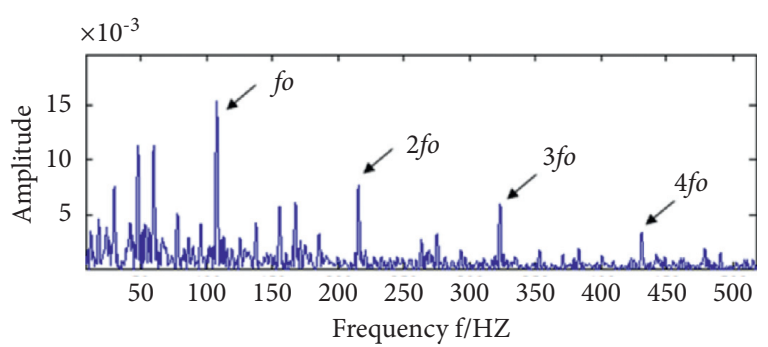

(b)

Figure 5: (a) Outer race fault signal; (b) envelope spectrum of outer race fault signal.

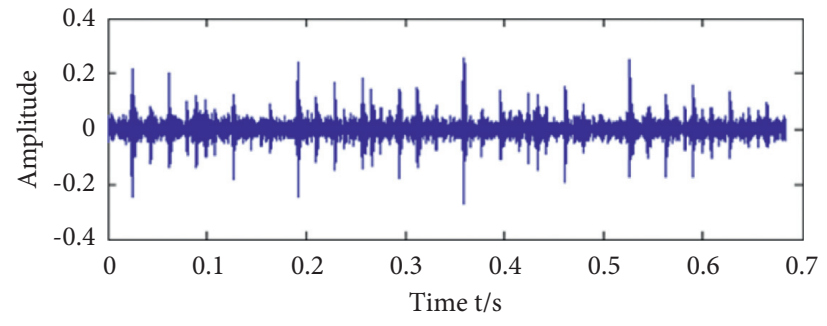

(a)

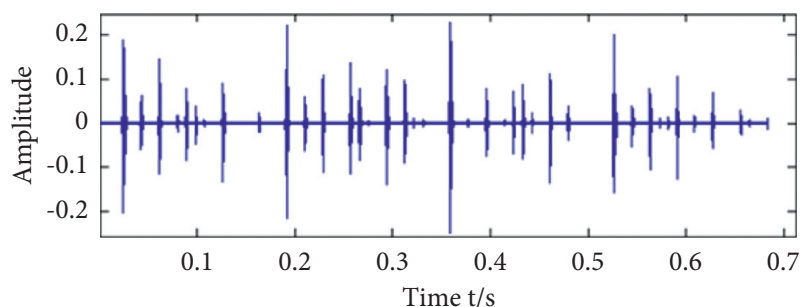

(b)

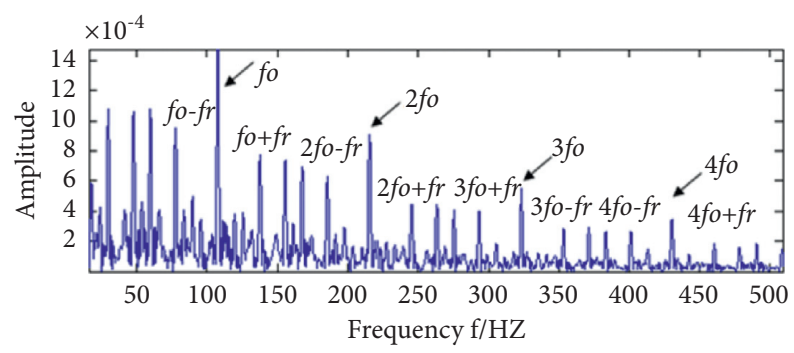

(c)

FiguRE 6: (a) Reconstructed signal of CWT; (b) sparse representation results by ASR; (c) envelope spectrum.

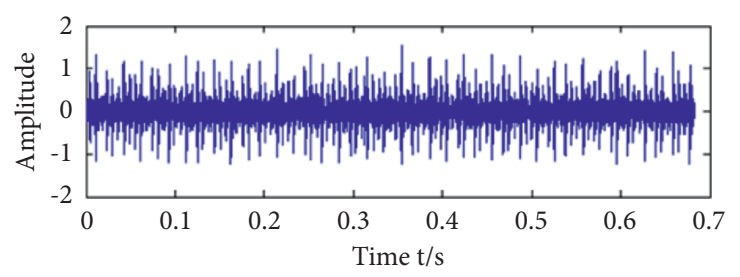

(a)

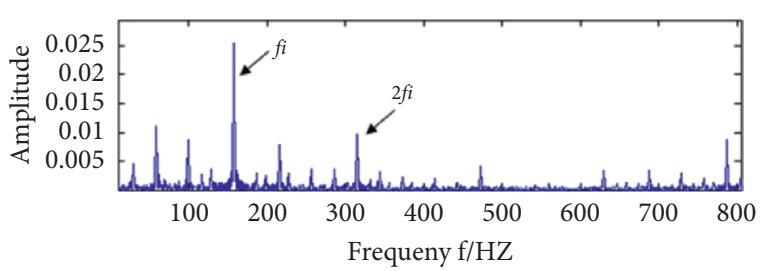

(b)

FIgURE 7: (a) Inner race fault signal; (b) envelope spectrum of inner race fault signal. 


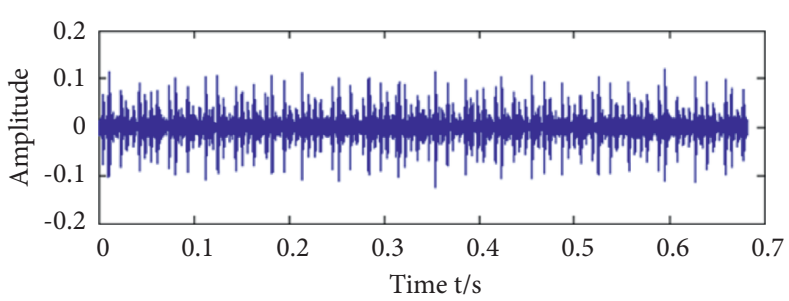

(a)

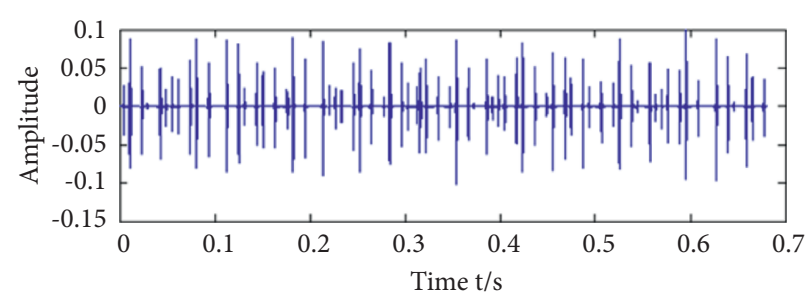

(b)

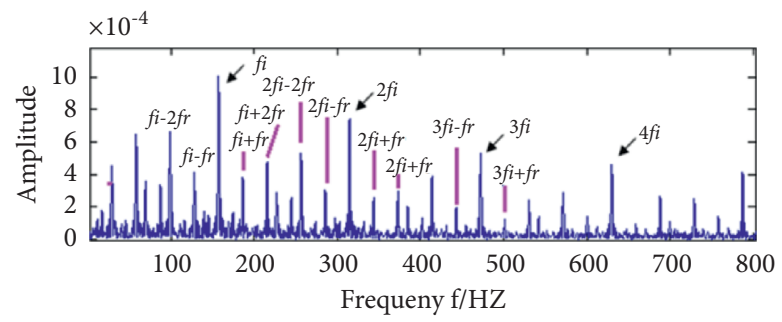

(c)

Figure 8: (a) Reconstructed signal of CWT; (b) sparse representation results by ASR; (c) envelope spectrum.

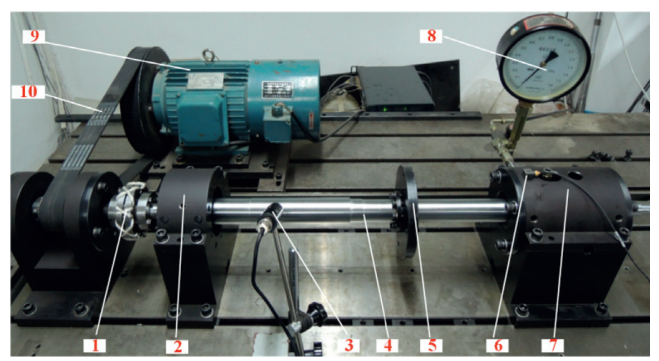

FIGURE 9: Rolling bearing test apparatus. 1. flexible coupling; 2. left bearing pedestal; 3. speed sensor; 4. Shaft; 5. mass disc; 6. Acceleration sensor; 7. right bearing pedestal; 8 . hydraulic gauge of preload display; 9. driving motor; 10. driving belt.

(Figure 7). Nevertheless, it reduces the magnitude of sideband frequencies, which may mislead the decisions in fault detection. The processing results of the proposed approach $(Q=1.6$ and $J=9)$ are shown in Figure 8. It can be seen that the fault frequencies $f i(162.18 \mathrm{~Hz}), 2 f i, 3 f, 4 f i$ and its sideband frequencies have been significantly enhanced, which is very important for bearing fault diagnosis.

5.2. Case Study 2. In order to further validate the reliability and superiority of the proposed method for fault diagnosis of rolling bearing, the data collected from the test apparatus were adopted to diagnose the bearing fault for analysis purposes. The test apparatus is shown in Figure 9, with the rotating speed of $240 \mathrm{rpm}$ and the sampling frequency of $48 \mathrm{~K}$. The test bearing is 7206 angular contact ball bearing, in which the number of balls is $Z=12$, ball diameter is $D_{b}=9.525 \mathrm{~mm}$, pitch circle diameter is $D_{p}=46 \mathrm{~mm}$, and contact angle is $\beta=25^{\circ}$. The original vibration signal of test bearing and its envelope spectrum are shown in Figure 10. It is difficult to find valuable characteristic information from the time domain waveform and its envelope spectrum due to the low speed and strong background noise. In particular, the energy excited by the bearing at low speed is often feeble and is not easily accessible for the impulse test. For the above reason, we apply the novel approach based on CWT and ASR for bearing fault detection.

The reconstructed signal of CWT is shown in Figure 11(a). As expected, the reconstructed signal eliminates a part of the noise interference, and the impulse characteristics are improved to a certain extent. Figure 11(b) displays the signal processed by $\operatorname{ASR}(Q=1.1$ and $J=9)$, and the impulse is further enhanced. This also indicates the ability to reveal the deeply hidden impulses and makes the proposed CWT-ASR approach more attractive for purifying weak mechanical fault signals. Moreover, the detailed envelope spectrum analysis results are shown in Figure 12. The fault frequencies $f i(28.5 \mathrm{~Hz}), 2 f i$, and its sideband frequencies $f i+f r, f i-f r, f i+2 f r, f i-2 f r, 2 f i+f r, 2 f i-f r$ can be clearly seen from the envelope spectra without being clouded by other peaks around them, and the faults can be detected accordingly: $27.24 \mathrm{~Hz}$ for the inner-race fault. Table 1 provides the frequency values corresponding to the envelope spectrum. The results also show that the proposed method has good feasibility in extracting periodic impulse signal and weak feature enhancement, and is suitable for the fault diagnosis of rolling bearing. 


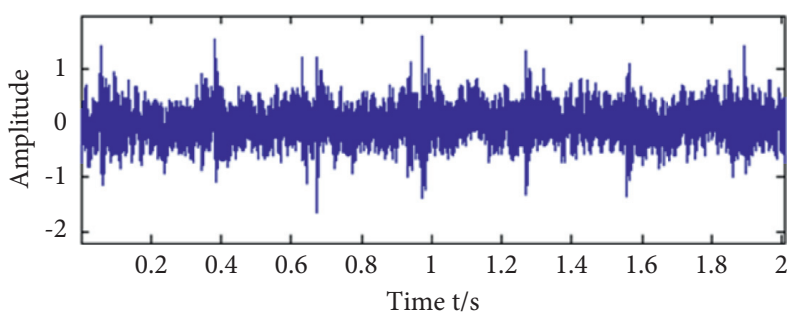

(a)

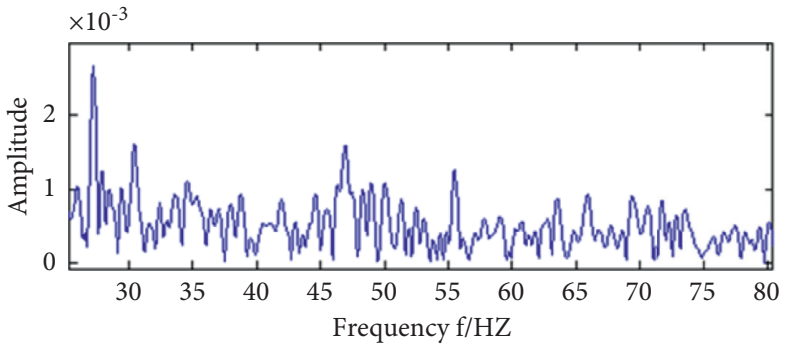

(b)

FIgURE 10: (a) Vibration signal of rolling bearing; (b) envelope spectrum.

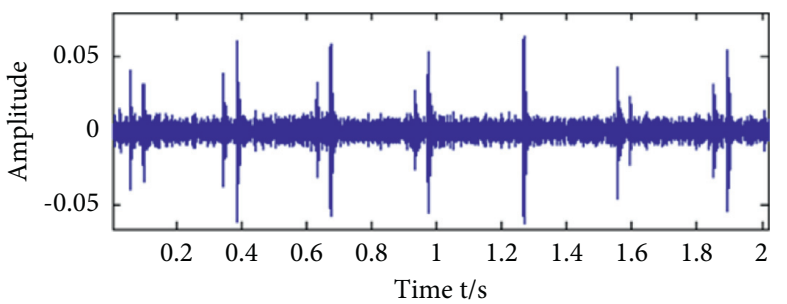

(a)

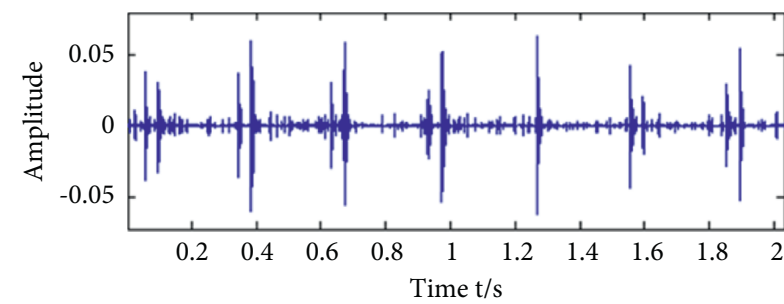

(b)

FIGURE 11: (a) Reconstructed signal of CWT; (b) sparse representation results by ASR.

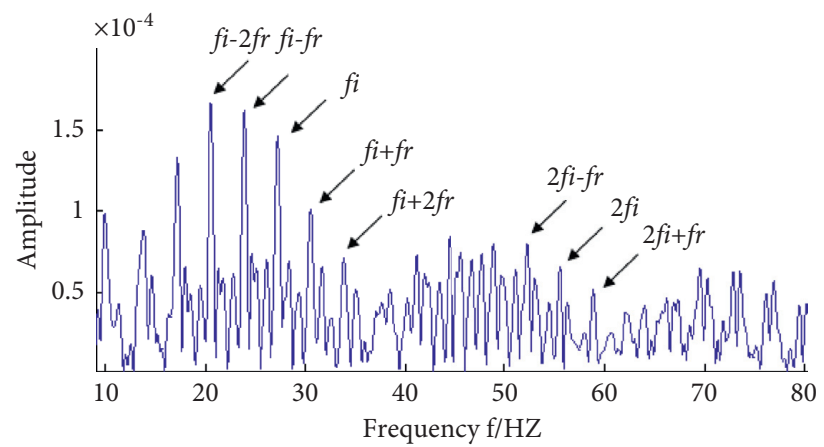

Figure 12: Envelope spectrum.

TABLE 1: Corresponding frequency values of envelope spectrum.

\begin{tabular}{lccccccc}
\hline$f i-2 f r(\mathrm{~Hz})$ & $f i-f r(\mathrm{~Hz})$ & $f i(\mathrm{~Hz})$ & $f i+f r$ & $f i+2 f r$ & $2 f i-f r(\mathrm{~Hz})$ & $2 f i(\mathrm{~Hz})$ & $2 f i+f r$ \\
\hline 20.6 & 23.9 & 27.24 & $30.53 \mathrm{~Hz}$ & $33.87 \mathrm{~Hz}$ & 52.28 & 55.53 & $58.87 \mathrm{~Hz}$ \\
\hline
\end{tabular}

\section{Conclusion}

In this work, a periodic impulse extraction method that combines the CWT and TQWT- SCS is proposed and investigated for the fault diagnosis of rolling bearing. The main conclusions of this article can be summarized into two aspects: (1) SCS algorithm is a feasible and convenient tool for signal sparse representation. Based on TQWT, this article proposes a new ASR method, which can perform sparse representation and enhance impulse signals efficiently. (2) An optimal scale selection scheme based on weighted Shannon entropy is proposed. CWT and TQWT constructed in the frequency domain are combined to give full play to their respective advantages and further enrich the application of wavelet theory in mechanical signal processing. Finally, the effectiveness and reliability of the proposed method are verified by simulation and real signals. 


\section{Data Availability}

All data included in this study are available upon request by contact with the corresponding author.

\section{Conflicts of Interest}

The authors declare no conflicts of interest with respect to the research, authorship, and/or publication of this article.

\section{Acknowledgments}

This work was financially supported by the Key-Area Research and Development Program of Guangdong Province (Grant no. 2020B090927002), the project of National Natural Science Funds of Shaanxi Province (Grant no. 2021JM017) and the National Natural Science Foundation of China (Grant no. 51975462).

\section{References}

[1] H. Cao, L. Niu, S. Xi, and X. Chen, "Mechanical model development of rolling bearing-rotor systems: a review," $\mathrm{Me}$ chanical Systems and Signal Processing, vol. 102, no. 1, pp. 37-58, 2018.

[2] R. B. Randall and J. Antoni, "Rolling element bearing diagnostics-A tutorial," Mechanical Systems and Signal Processing, vol. 25, no. 2, pp. 485-520, 2011.

[3] N. Sawalhi, R. B. Randall, and H. Endo, "The enhancement of fault detection and diagnosis in rolling element bearings using minimum entropy deconvolution combined with spectral kurtosis," Mechanical Systems and Signal Processing, vol. 21, no. 6, pp. 2616-2633, 2007.

[4] J. Antoni and R. B. Randall, "The spectral kurtosis: application to the vibratory surveillance and diagnostics of rotating machines," Mechanical Systems and Signal Processing, vol. 20, no. 2, pp. 308-331, 2006.

[5] J. Antoni, "The spectral kurtosis: a useful tool for characterising non-stationary signals," Mechanical Systems and Signal Processing, vol. 20, no. 2, pp. 282-307, 2006.

[6] J. Antoni, F. Bonnardot, A. Raad, and M. E. Badaoui, "Cyclostationary modelling of rotating machine vibration signals," Mechanical Systems and Signal Processing, vol. 18, no. 6, pp. 1285-1314, 2004.

[7] I. Antoniadis and G. Glossiotis, "Cyclostationary analysis of rolling-element bearing vibration signals," Journal of Sound and Vibration, vol. 248, no. 5, pp. 829-845, 2001.

[8] C. Peeters, J. Antoni, and J. Helsen, "Blind filters based on envelope spectrum sparsity indicators for bearing and gear vibration-based condition monitoring," Mechanical Systems and Signal Processing, vol. 138, Article ID 106556, 2020.

[9] Z. Zhang and J. Zhong, "Multi-fault diagnosis for rolling element bearings based on ensemble empirical mode decomposition and optimized support vector machines," $M e$ chanical Systems and Signal Processing, vol. 41, no. 1-2, pp. 127-140, 2013.

[10] R. Yan, R. X. Gao, and X. Chen, "Wavelets for fault diagnosis of rotary machines: a review with applications," Signal Processing, vol. 96, pp. 1-15, 2014.

[11] J. Lin, M. J. Zuo, and K. R. Fyfe, "mechanical fault detection based on the wavelet de-noising technique," Journal of Vibration and Acoustics, vol. 126, no. 1, pp. 9-16, 2004.
[12] J. Lin and L. Qu, "Feature extraction based on morlet wavelet and its application for mechanical fault diagnosis," Journal of Sound and Vibration, vol. 234, no. 1, pp. 135-148, 2000.

[13] A. Silva, A. Zarzo, J. M. Machuca González, and J. M. MunozGuijosa, "Early fault detection of single-point rub in gas turbines with accelerometers on the casing based on continuous wavelet transform," Journal of Sound and Vibration, vol. 487, Article ID 115628, 2020.

[14] I. W. Selesnick, "Wavelet transform with tunable Q-factor," IEEE Transactions on Signal Processing, vol. 59, no. 8, pp. 3560-3575, 2011.

[15] J. Luo, D. Yu, and M. Liang, "A kurtosis-guided adaptive demodulation technique for bearing fault detection based on tunable-Q wavelet transform," Measurement Science and Technology, vol. 24, no. 5, Article ID 055009, 2013.

[16] W. He, Y. Zi, B. Chen, S. Wang, and Z. He, "Tunable Q-factor wavelet transform denoising with neighboring coefficients and its application to rotating machinery fault diagnosis," Science China Technological Sciences, vol. 56, no. 8, pp. 1956-1965, 2013.

[17] I. W. Selesnick, "Resonance-based signal decomposition: a new sparsity-enabled signal analysis method," Signal Processing, vol. 91, no. 12, pp. 2793-2809, 2012.

[18] G. Cai, I. W. Selesnick, S. Wang, W. Dai, and Z. Zhu, "Sparsity-enhanced signal decomposition via generalized minimax-concave penalty for gearbox fault diagnosis," Journal of Sound and Vibration, vol. 432, pp. 213-234, 2018.

[19] G. Cai, X. Chen, and Z. He, "Sparsity-enabled signal decomposition using tunable Q-factor wavelet transform for fault feature extraction of gearbox," Mechanical Systems and Signal Processing, vol. 41, no. 1-2, pp. 34-53, 2013.

[20] D. Zhang and D. Yu, "multi-fault diagnosis of gearbox based on resonance-based signal sparse decomposition and comb filter," Measurement, vol. 103, pp. 361-369, 2017.

[21] S. Mallat, A Wavelet Tour of Signal Processing, Second Edition, Academic Press, Cambridge, Massachusetts, 1999.

[22] A. Hyvrinen, "Sparse code shrinkage: denoising of nongaussian data by maximum likelihood estimation," Neural Computation, vol. 11, no. 7, pp. 1739-1768, 1999.

[23] D. L. Donoho, "De-noising by soft-thresholding," IEEE Transactions on Information Theory, vol. 41, no. 3, pp. 613-627, 1995.

[24] D. L. Donoho and I. M. Johnstone, "Ideal spatial adaptation by wavelet shrinkage," Biometrika, vol. 81, no. 3, pp. 425-455, 1994.

[25] Z. Mo, J. Wang, H. Zhang, and Q. Miao, "Weighted cyclic harmonic-to-noise ratio for rolling element bearing fault diagnosis," IEEE Transactions on Instrumentation and Measurement, vol. 69, no. 2, pp. 432-442, 2020.

[26] Q. Ni, J. C. Ji, K. Feng, and B. Halkon, “A fault informationguided variational mode decomposition (FIVMD) method for rolling element bearings diagnosis," Mechanical Systems and Signal Processing, vol. 164, Article ID 108216, 2022.

[27] https://engineering.case.edu/bearingdatacenter. 